\title{
Research And Implementation Of Geometric Correction Algorithm For Spatial Target Image Recognition
}

\author{
Hongyan Chen, Junwei Wan \\ Beijing Institute of Tracking and Telecommunicaitons Technology,Beijing 100094, China \\ child_smile@163.com
}

\begin{abstract}
Keywords: Space object recognition; image processing; geometric correction; Remote Sensing and Telemetry
\end{abstract}

Abstract. In the process of space target surveillance system to obtain the target image in space, due to the impact of imaging equipment is limited and some other random factors, Therefore, the final image is blurred and distorted, so it is difficult to estimate and track the target in the later stage. In this paper, based on the geometric correction technique of space target images, Put forward through the study of spatial transformation, gray interpolation and unknown variation algorithm to construct a mapping model,In the image preprocessing part, the distorted image is corrected, To obtain more accurate target centroid coordinates and clearer target images, the high accuracy of the space target surveillance system is achieved.

\section{Introduction}

In the process of obtaining space target images by remote sensing monitoring, Because of the limitation of the imaging device and some other random factors, the final image is blurred and distorted. Therefore, only by eliminating the influence of these factors can the image of the space target be restored with high resolution. The geometric correction of space object images is one of the key technologies, which is related to the accuracy of the whole space target surveillance system.

\section{Existing problems analysis}

Because the structure of the optical lens is different from the ideal pinhole model, The height of the intersection of the primary ray and Gauss image does not equal the height of the ideal image, a pair of images and objects, The vertical magnification varies with the angle of view field, so that the actual coordinates of the pixels in the image are not consistent with the corresponding theoretical coordinates, which is the error caused by nonlinear distortion.

Specifically, geometric distortion is the combined effect of translation, scaling, rotation, affine, partial twisting, bending, and higher fundamental deformations, and the process of eliminating such differences is called Geometric Correction. Images acquired in natural environment, because of the imaging system itself and some external reasons, the image will suffer geometric distortion and skew deformation, Therefore, the target information in the image will also be distorted, which brings the difficulty of image analysis, target identification and tracking in the later stage. Such as space and space photography, the earth's equidistant parallel lines, the image will be skewed, or parallel, but unequal spacing. As shown in Figure 1.

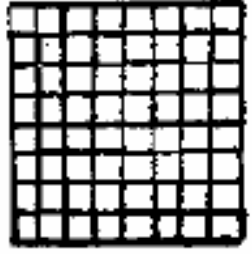

(a) original image
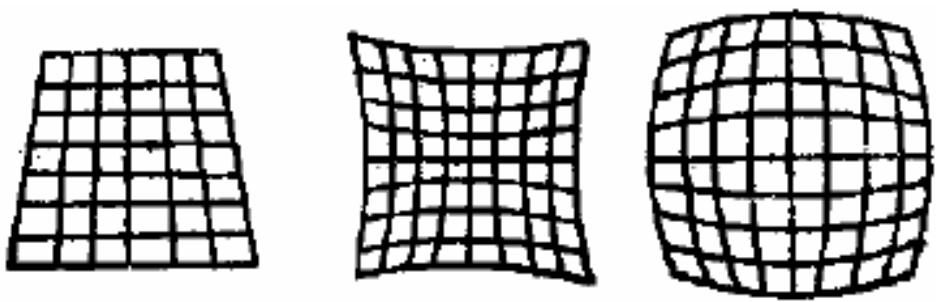

(b) (c) (d) distorted image

Fig.1 Distortion image example 
The image distortion will inevitably bring about unpredictable influence on the target segmentation and target recognition in the later stage. Therefore, it is necessary to correct the distorted image in the image preprocessing section, The location of each pixel in the distorted image is corrected by geometric transformation, and the original spatial relation and gray relation between pixels are re obtained.

\section{Main algorithm research}

In the process of remote sensing monitoring, the influence of the external space interference and the load noise in the imaging equipment will lead to geometric distortion of the target in the CCD probe response. In order to get a more accurate target centroid coordinates, based on star catalogue geometric correction is very important. In image preprocessing, geometric correction is also an essential part of obtaining clearer target images. Technically, the research of the algorithm has the following difficulties:

1) The establishment of the corresponding relationship between image and star catalogue;

2) Effective selection of control points: When the human intervention control point is selected, the influence of the adult and the distribution of the human's own point of view will also affect the correction effect; The automatic corner detection technology has certain scope of application, and it is not possible to select the most representative control points;

3) Determination of mapping model: Since the correlation parameters of imaging equipment are uncertain, How to choose a more accurate linear or nonlinear model to describe the mapping between the distorted image and the standard image is a difficult point of the algorithm.

The two main steps of the geometric correction problem are spatial transformation and gray scale interpolation. Geometric correction can be divided into two cases: the known transformation relation and the unknown transformation relation. When the transformation relation is known, spatial transformation and gray interpolation can be carried out directly; When the transform relation is unknown, it is usually necessary to select the control point method to determine the transform relation, and then the space transformation and gray interpolation are used to complete the geometric correction.

known transformations.Image space transformation means that one image $g(x, y)$ will be used to correct another image $f(u, v)$. According to the corresponding points of two images, a function relation is established, and the input image coordinate ${ }^{(u, v)}$ is changed to the output image coordinate $(x, y)$.

\section{1) Translation transformation}

In the two-dimensional coordinate system, point $P(x, y)$ is shifted ${ }^{t}{ }_{x}$ and ${ }^{t}{ }$ in the $\mathrm{X}$ axis and the $\mathrm{Y}$ axis respectively. As shown in Figure 2 (a), get a point $P^{\prime}\left(x^{\prime}, y^{\prime}\right)$, Then the coordinates of points $P$ and $P^{\prime}$ satisfy the relation:

$$
\left\{\begin{array}{l}
x^{\prime}=x+t_{x} \\
y^{\prime}=y+t_{y}
\end{array}\right.
$$

The vector expression is:

$$
P^{\prime}=P+T
$$




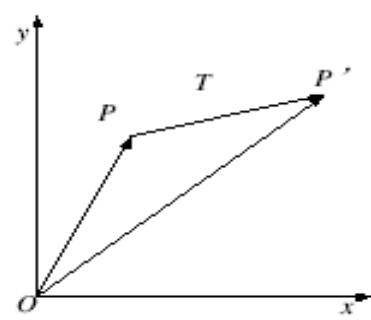

(a) Translation transformation

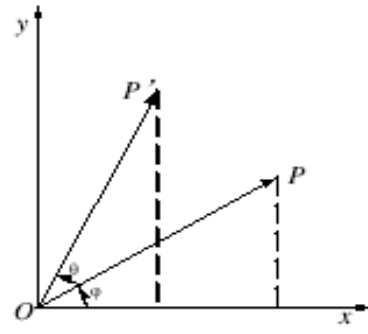

(b) Rotation transformation

Fig.2 Transform diagram

2) Rotation transformation

Given a fixed point $P(x, y)$, whose polar coordinates are in the form of:

$\left\{\begin{array}{l}x=r \cos \phi \\ y=r \sin \phi\end{array}\right.$

As shown in Figure $2(\mathrm{~b})$, rotate it around the origin of the coordinate angle $\theta$, get a point $P^{\prime}\left(x^{\prime}, y^{\prime}\right)$, then the coordinate relation between point $P^{\prime}$ and point $P$ is:

$\left\{\begin{array}{l}x^{\prime}=r \cos (\phi+\theta)=r \cos \phi \cos \theta-r \sin \phi \sin \theta=x \cos \theta-y \sin \theta \\ y^{\prime}=r \sin (\phi+\theta)=r \cos \phi \sin \theta+r \sin \phi \cos \theta=x \sin \theta+y \sin \theta\end{array}\right.$

3) Homogeneous coordinates and compound transformations

In the above transformation, the translation transformation is a vector addition, while the rotation scaling is transformed into matrix multiplication.In order to avoid the combination of expressions in combinatorial transformations, the synthesis of transformations is easier, need to be converted to homogeneous coordinates. For the complex transformation of the image, the complex transformation is first decomposed into a number of basic transformations, and then applied to the image, so that it is more intuitive and image.

Gray-Level Interpolation.After the space coordinate transformation, the gray value of each pixel point in the target image must be determined. The gray value of image pixel is generally divided into two steps: gray interpolation and gray mapping.

1) Nearest neighbor interpolation

Nearest neighbor interpolation is a simple interpolation algorithm, also known as zero order interpolation. For a floating point coordinates obtained by an inverse transform, simply rounding the (or four to five homes), get an integer integer coordinates, the coordinates of the pixel gray value of the target pixel value is gray. Direct rounding, the coordinates of the corresponding angle on the floating point pixel value; four to five homes, then take the distance floating point coordinates nearest point pixel value. As shown in figure 3.

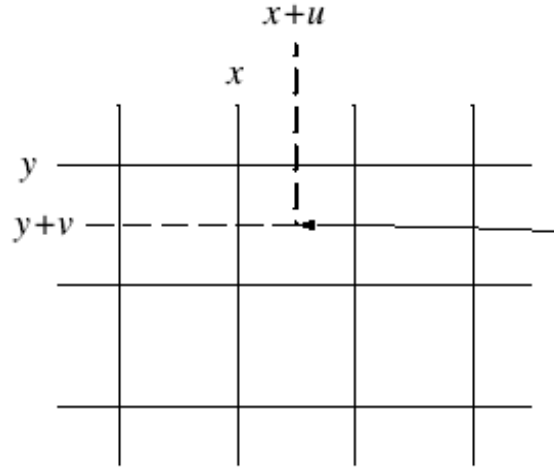

(a) original

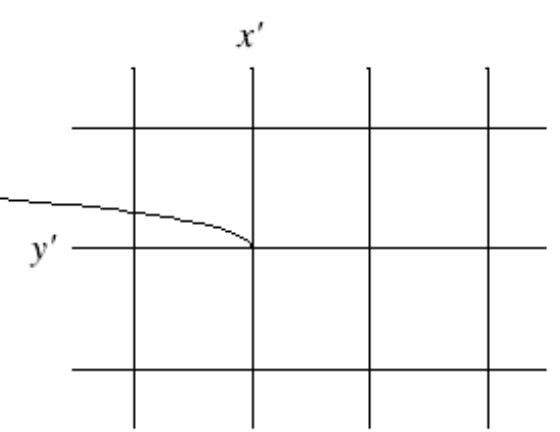

(b) object

Fig.3 Sketch map of nearest neighbor interpolation 
2) Bilinear interpolation

Bilinear interpolation algorithm, also known as the first order interpolation algorithm, its effect is better than the nearest neighbor interpolation algorithm. As shown in Figure 4, assume $0<x<1,0<y<1$.

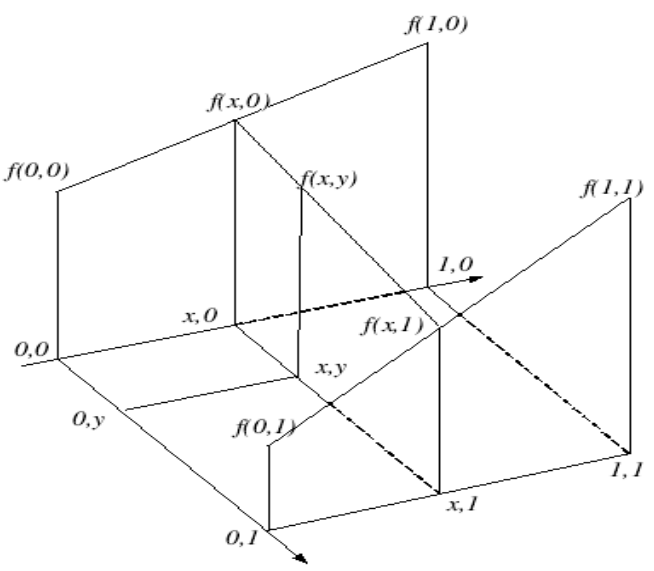

Fig.4 Schematic diagram of bilinear interpolation

First, the first order linear interpolation is obtained, $f(x, 0)$ and $f(x, 1)$.Then, $f(x, 0)$ and $f(x, 1)$ are used to perform first-order linear interpolation in the vertical direction to determine $f(x, y)$. Formula for:

$$
\begin{aligned}
& f(x, y)=x[f(1,0)-f(0,0)]+y[f(0,1)-f(0,0)]+ \\
& x y[f(1,1)+f(0,0)-f(0,1)-f(1,0)]+f(0,0)
\end{aligned}
$$

The bilinear interpolation algorithm has large computation, but the processed image quality is high,and no pixel values are discontinuous. Because the bilinear interpolation algorithm has the property of low pass filter and the high frequency component is damaged, the contour of the image will be blurred to a certain extent.

3) Gray value mapping

There are two ways to map grayscale values, as shown in figure 5 .
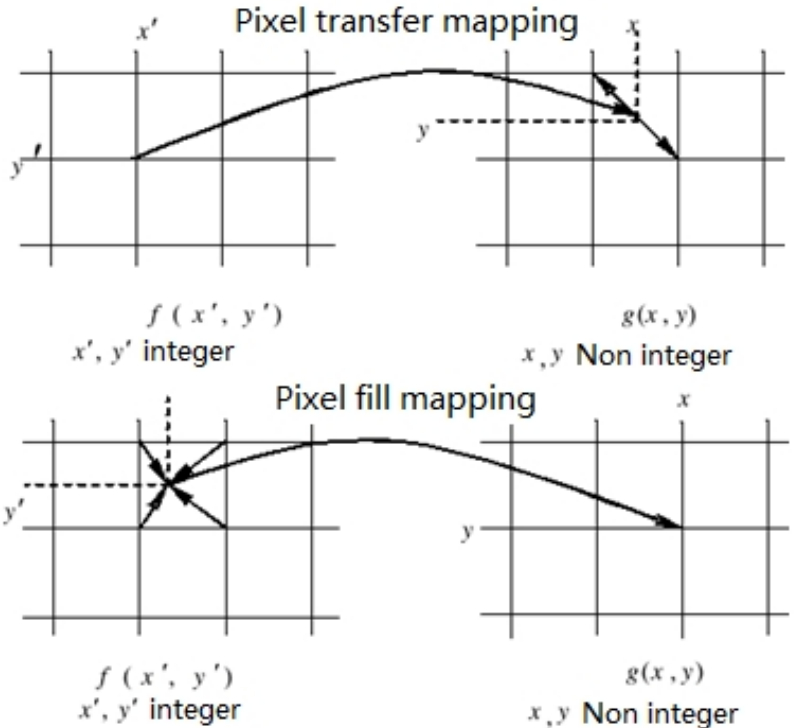

Fig.5 Gray value mapping

One is to think of geometric operations as transferring pixels of an input image one pixel at a time to an output image. If an input pixel is mapped to the position between four output pixels, its gray value is allocated between four output pixels according to the interpolation algorithm. The method is called forward mapping, also called pixel transfer mapping. 
The other is the backward mapping method, also called pixel fill mapping. The output pixels are mapped back to the input image one by one in order to determine their gray levels. If an output pixel is mapped between four input pixels, its gray value is determined by gray level interpolation.

Unknown transformation. When the unknown transform relation is concerned, the transformation relation must be determined first, and then the geometric correction is completed according to the method mentioned in the known transformation relation. To determine the change relationship, first the mapping model should be assumed, and then the relevant parameters are determined. In the process of determining the parameters, through the corner detection technology and control points matching, find the appropriate corner, and then determine the relevant parameters. When choosing the mapping model, we can choose the two polynomial model in general. If it is linear distortion, then the affine transformation model can be selected.

1) Manually select control points

The process of manually selecting a control point is equivalent to the corner detection and control point matching in the automatic selection of control points. In selecting the control point manually, the following conditions should be met for the selected points: In the standard image and the distorted image, the selected control points should be one-to-one correspondence, so that the matching process can be omitted; The selected control points on the image should be distributed as evenly as possible; The selected control points on the image should best reflect the law of distortion.

2) Control point matching

The commonly used matching methods include relaxation method, relative distance histogram, aggregation beam detection method, Hausdorff distance and correlation method and so on. However, these methods are very demanding on the detected corner points, such as requiring the same number, simple transformation relations, and so on, and therefore can not meet the general requirements. We adopt global matching and compute similarity to select the closest pairs of control points.

3) Mapping relation

The geometric distortion of the image taken by the camera is obviously nonlinear, so the nonlinear polynomial is used to fit the mapping relation, which is usually expressed by polynomial transformation between coordinates, and the least square sum of errors is fitted. Similarly, for linear distortion modes, Affine mapping can be used to fit mapping relations, which can be regarded as translation transformations, scale transformations, rotation transformations, and complex transformations of cut and wrong transformations.

\section{Summary}

A large number of experiments show that the proposed algorithm combines spatial variation, gray level interpolation and unknown transform, In a variety of space environment, the target recognition rate has been greatly improved after geometric correction.

With the development of aerospace technology and remote sensing technology, people can monitor space targets more accurately and faster. Especially in military field, remote sensing and processing technology have been paid more attention to. The accurate target monitoring, analysis and evaluation of remote sensing images have been integrated with the long-range attack and support system of aircraft. With the ability to obtain the space surveillance system to air observation data increasing, how to achieve high precision of the image data to spatial target information acquisition geometry accurate processing, is the basis of effective space surveillance of air observation advantage. Therefore, the research on the theory and method of high-precision geometric correction of remote sensing images, providing more accurate application potential of target centroid coordinates, will be urgent and of great practical significance. 


\section{References}

[1] Analysis and quantification of laser-dazzling effects on IR focal plane arrays. Hueber N,Vincent D,Morin A,et al. Proceedings of SPIE on Infrared Technology and Applications XXXVI . 2010 [5]

[2] Robust Watermarking Algorithm for Digital Image Based on SIFT Feature Points. ZOU Xin-guo,LI Na,NAWEI J. Software Engineering and Service Science(ICSESS) 2014 5th IEEE International Conference on . 2014

[3] Survey-only Optical Strategies for Cataloguing Space Debris Objects in the Future European Space Surveillance System. Olmedo E,Sannchez-Ortiz N,Guijarro N,et al. Advances in Space Research . 2011 [8]

[4] A Debris Image Tracking Using Optical Flow Algorithm. Fujita K,Hanada T,Kitazawa Y,et al. Advances in Space Research . 2012

[5] Parallel implementation of Gray Level Co-occurrence Matrices and Haralick texture features on cell architecture $[\mathrm{J}]$. Asadollah Shahbahrami,Tuan Anh Pham,Koen Bertels. The Journal of Supercomputing . 2012 (3)

[6] A Semi Blind DWT-SVD Video Watermarking[J] . Divjot Kaur Thind,Sonika Jindal. Procedia Computer Science . 2015

[7] Effect of the sampling design of ground control points on the geometric correction of remotely sensed imagery[J] . Jianghao Wang,Yong Ge,Gerard B.M. Heuvelink,Chenghu Zhou,Dick Brus. International Journal of Applied Earth Observations and Geoinformation . 2012

[8] Line-based multi-label energy optimization for fisheye image rectification and calibration. Zhang Mi,Yao Jian,Xia Menghan,et al. 2015 IEEE Conference on Computer Vision and Pattern Recognition (CVPR) . 2015

[9] Fast and robust perspective rectification of document images on a smartphone. WILIEM P,SIMON C,CHO S,et al. IEEE Conference on Computer Vision and Pattern Recognition Workshops . 2014

[10] Assessing geometric accuracy of the orthorectification process from GeoEye-1 and WorldView-2 panchromatic images[J] . Manuel A. Aguilar,María del Mar Salda?a,Fernando J. Aguilar. International Journal of Applied Earth Observations and Geoinformation . 2013 